\title{
Infrared detection of gas phase formaldehyde towards the high mass protostar W33A
}

\author{
E. Roueff ${ }^{1}$, E. Dartois ${ }^{2}$, T. R. Geballe ${ }^{3}$, and M. Gerin ${ }^{4}$
}

\author{
${ }^{1}$ Laboratoire Univers et Théorie, UMR 8102 du CNRS, Observatoire de Paris, Section de Meudon, Place Jules Janssen, 92195 Meudon, France \\ e-mail: evelyne.roueff@obspm.fr \\ 2 IAS, Bat. 121, Université Paris Sud, 91405 Orsay Cedex, France \\ 3 Gemini Observatory, 670 N. A'ohoku Place, Hilo, HI 96720, USA \\ 4 Laboratoire d'Étude du Rayonnement et de la Matière en Astrophysique, UMR 8112 du CNRS, Observatoire de Paris \\ and École Normale Supérieure, 24 rue Lhomond, 75231 Paris Cedex 05, France
}

Received 16 June 2005 / Accepted 17 October 2005

\section{ABSTRACT}

We report the detection of numerous absorption lines of the $v_{1}$ band of gaseous formaldehyde $\left(\mathrm{H}_{2} \mathrm{CO}\right)$ near $3.6 \mu \mathrm{m}$ towards the high-mass protostar W33A. This is the first infrared detection of gaseous $\mathrm{H}_{2} \mathrm{CO}$ in an interstellar cloud. An upper limit toward RAFGL 7009S is also reported. The column density of $\mathrm{H}_{2} \mathrm{CO}$ detected at $3.6 \mu \mathrm{m}$ toward $\mathrm{W} 33 \mathrm{~A}$ is much higher than that measured in large beam millimeter wave observations toward the same source, suggesting that it is concentrated close to the infrared continuum source. The mean temperature of the $\mathrm{H}_{2} \mathrm{CO}$, derived from LTE spectral modelling, is $\sim 100 \mathrm{~K}$, which is close to the values found for most other gas phase species observed in absorption and is the expected temperature of the cloud at the dust mantle sublimation interface, implying a large jump in the abundance of gaseous $\mathrm{H}_{2} \mathrm{CO}$ from the surrounding cold cloud to the warm gas. The ratio of gaseous and solid $\mathrm{H}_{2} \mathrm{CO}$ column densities is about 0.03 for W33A and less than 0.02 for RAFGL 7009S. We discuss this detection in the context of the two most likely mechanisms for $\mathrm{H}_{2} \mathrm{CO}$ formation: grain mantle evaporation, and gas phase reactions between species produced by photodissociation of grain mantle evaporants.

Key words. ISM: molecules - molecular processes - stars: circumstellar matter - stars: formation

\section{Introduction}

Ground-based high resolution $\left(R>10^{4}\right)$ infrared spectroscopy of molecular vibration-rotation bands toward massive protostars is capable of probing a wide range of environments around these objects. In many cases it provides access to hot regions, close to the star but just outside the ionization zone, where molecules barely survive and where grains have evaporated (Mitchell et al. 1990; Boogert et al. 2004). It also almost always reveals the more distant and cold regions of the starforming cloud, where the molecules and dust grains are as yet unaffected by events in the cloud cores. In some cases infrared spectroscopy of key trace molecules can probe an important interface between these two regions, where grain mantles, which formed when the cloud was cold, are evaporating.

We present in this paper an infrared detection of gas phase formaldehyde $\left(\mathrm{H}_{2} \mathrm{CO}\right)$ at $3.6 \mu \mathrm{m}$ toward the high mass protostar W33A, obtained using high resolution absorption spectroscopy, and argue that much of the $\mathrm{H}_{2} \mathrm{CO}$ we have detected is either newly evaporated from grains or has formed from the products of the dissociation of recently evaporated species. The observations are described in the next section. The infrared spectroscopic properties of $\mathrm{H}_{2} \mathrm{CO}$ are summarized in Sect. 3. Results are given in Sect. 4; these include simulated spectra of $\mathrm{H}_{2} \mathrm{CO}$ at different temperatures. We then discuss the results in relation to the abundances and composition of ice mantles which have been measured by ISO (Infrared Space Observatory) in deeply embedded massive young stars (as summarized in Gibb et al. 2004). Section 6 contains our conclusions.

\section{Observations}

High resolution spectra of two protostars in dense cloud cores, W33A and RAFGL 7009S (coordinates and radial velocities given in Table 1), were obtained in photometric and stable weather conditions at the UK $3.8 \mathrm{~m}$ Infrared Telescope (UKIRT) on UT 2004 June 9, using the facility spectrometer CGS4 (Mountain et al. 1990). The instrument was configured with an echelle tuned to cover $2736-2785 \mathrm{~cm}^{-1}$ $(3.59-3.65 \mu \mathrm{m})$ and a $0.45^{\prime \prime}$ wide slit which provided a resolving power of 33000 . Spectra were sampled every $1 / 3$ resolution element by physically translating the array in the dispersion direction after individual $15 \mathrm{~s}$ exposures, and were obtained with the astronomical source alternatively positioned at two locations along the slit. W33A was observed for a total of $36 \mathrm{~min}$ and RAFGL 7009S was observed for $42 \mathrm{~min}$. The bright star HR 6378 (A2V) was observed for the purposes of flux 
Table 1. Positions and velocities of the observed sources.

\begin{tabular}{lrrr}
\hline \hline Source & $\begin{array}{r}\text { RA } \\
\mathrm{J} 2000\end{array}$ & $\begin{array}{r}\text { Dec } \\
\mathrm{J} 2000\end{array}$ & $\begin{array}{r}V_{\mathrm{LSR}} \\
\mathrm{km} \mathrm{s}^{-1}\end{array}$ \\
\hline W33A & $18: 14: 39.9$ & $-17: 51: 59$ & $31^{a}$ \\
RAFGL 7009S & $18: 34: 20.91$ & $-05: 59: 42.2$ & $41.5^{b}$ \\
\hline
\end{tabular}

${ }^{a}$ McCall et al. (1999); ${ }^{b}$ Bronfman et al. (1996).

calibration and removal of telluric lines; its average airmass during the observation matched those of both W33A and RAFGL 7009S to better than 2 percent. As the strongest telluric lines were 40 percent deep and most were much weaker, the depths of all telluric lines in the target and calibration spectra should be and in fact were observed to match to better than one percent.

Data reduction was standard, consisting of coaddition of pairs of subtracted frames, extraction of one-dimensional spectra from the coadded frame, removal of spikes, wavelength calibration (which used telluric lines and is accurate at $2 \sigma$ to $0.09 \mathrm{~cm}^{-1}$ or $0.00012 \mu \mathrm{m}$. This was followed by cross correlation and slight wavelength shifting of the target spectrum so that its telluric lines matched those in the spectrum of the calibration star. Division of the target spectrum by the standard spectrum and flux calibration were then carried out. The resulting spectra are displayed in Fig. 1.

The observing program originally was aimed at detecting $\mathrm{D}_{2} \mathrm{H}^{+}$which has a strong transition near $2736 \mathrm{~cm}^{-1}$, and $\mathrm{H}_{2} \mathrm{D}^{+}$ at other frequencies, in sources in which $\mathrm{H}_{3}^{+}$is known or suspected to be abundant (Geballe \& Oka (1996). No transitions corresponding to $\mathrm{D}_{2} \mathrm{H}^{+}$were detected (Roueff et al., in preparation). However, the spectrum towards W33A displayed numerous unexpected absorption lines, which we identified as belonging to the $v_{1}$ band of formaldehyde $\left(\mathrm{CH}_{2}\right.$ symmetric stretch). This band has been seen previously in the spectra of a few comets (Knacke et al. 1986; Mumma \& Reuter 1989; Disanti et al. 2002) but never before in the interstellar medium. Figure 1 shows the fully reduced spectra towards W33A and RAFGL 7009S in the 2780-2795 $\mathrm{cm}^{-1}$ band together with a simulated spectrum of $\mathrm{H}_{2} \mathrm{CO}$ computed for a column density of $2 \times 10^{16} \mathrm{~cm}^{-2}$ and a temperature of $90 \mathrm{~K}$ (further details given in Sect. 4), which gives a reasonably good fit to the W33A spectrum.

Formaldehyde is sometimes present at very low levels (ppbv) in the atmosphere. However, any telluric $\mathrm{H}_{2} \mathrm{CO}$ lines would be very weak above Mauna Kea, would be shifted relative to those in $\mathrm{W} 33 \mathrm{~A}$, and would also divide out during data reduction as did much stronger lines due to telluric $\mathrm{CH}_{4}$ and $\mathrm{H}_{2} \mathrm{O}$. The above arguments, the multitude of identified $\mathrm{H}_{2} \mathrm{CO}$ lines in W33A, the acceptable match of the spectrum to a much lower than telluric temperature, and the absence of $\mathrm{H}_{2} \mathrm{CO}$ in RAFGL 7009S which was observed nearly simultaneously with W33A and reduced using the same telluric calibration star spectrum, put the reality of this first infrared detection of interstellar gaseous $\mathrm{H}_{2} \mathrm{CO}$ beyond question.

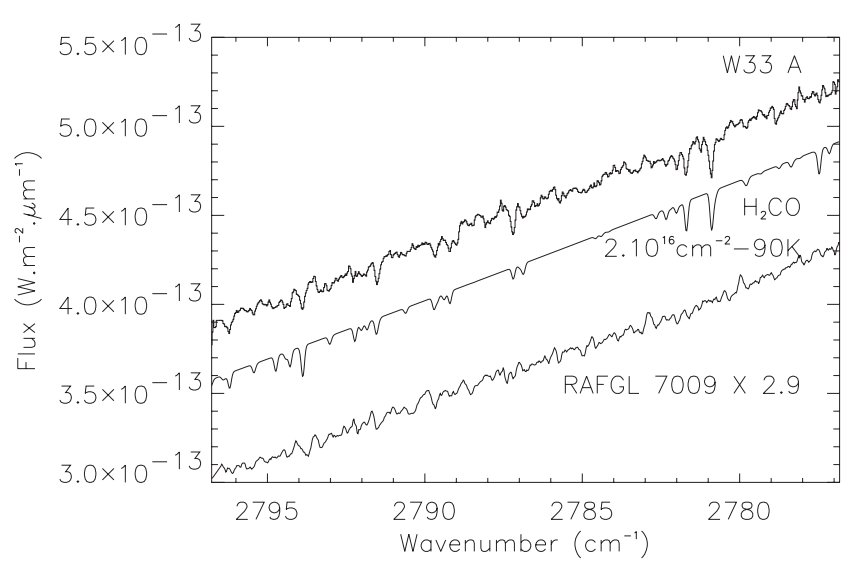

Fig. 1. UKIRT spectra of W33A and RAFGL 7009S compared to a gas phase $\mathrm{H}_{2} \mathrm{CO}$ modelled spectrum multiplied by the estimated continuum of W33A and slightly shifted down for clarity.

\section{The infrared spectrum of $\mathrm{H}_{2} \mathrm{CO}$}

\subsection{Gas phase}

Gas phase $\mathrm{H}_{2} \mathrm{CO}$ possesses six vibrational infrared active modes. The two strongest modes are the $v_{1}$ and $v_{5}$ stretches producing bands centered at $2782 \mathrm{~cm}^{-1}$ and $2843 \mathrm{~cm}^{-1}$ with a combined integrated intensity of about $648 \mathrm{~cm}^{-2} \mathrm{~atm}^{-1}$ (Herndon et al. 2005). The next most intense mode is the carbonyl vibration $v_{2}$ at $1742 \mathrm{~cm}^{-1}$ with an intensity of about $294 \mathrm{~cm}^{-2} \mathrm{~atm}^{-1}$ (Perrin et al. 2003). The stretching modes have been modelled using line intensities provided in the latest version of the HITRAN data base Rothman et al. (2003) which are derived from the previous assignments of Brown et al. (1979). Equal intensities were attributed to blended lines in Brown et al. (1979), which is questionable in some cases (A. Perrin, private communication). However, often the overlapping transitions arise from lower levels of widely different energies, in which case the propagated error in the simulated spectra at low rotational temperatures is reduced as the transition from the lowest energy dominates.

Both the $v_{2}$ band and the combined $v_{1}$ and $v_{5}$ bands are in the spectral range covered by ISO SWS. The resolution of ISO SWS is too low to detect individual ro-vibrational transitions, but in principle the Q-branches could be detected. A recent analysis of the $v_{2}, v_{3}, v_{4}$ and $v_{6}$ bands of $\mathrm{H}_{2} \mathrm{CO}$ by Perrin et al. (2003) has allowed an accurate determination of the hamiltonian and a precision computation of the intensities of individual transitions for these four bands. From the spectrum in Keane et al. (2001) we estimate that the upper limit on the $\mathrm{H}_{2} \mathrm{CO}$ column density in W33A (constrained principally via the pileups of lines at the $v_{2} \mathrm{Q}$ branches) is twice the value detected at UKIRT.

\subsection{Solid phase}

Maier et al. (1980) studied different vibrational modes of formaldehyde trapped in solid xenon at $15 \mathrm{~K}$ and showed that the $v_{2} \mathrm{CO}$ stretch $\left(1727 \mathrm{~cm}^{-1}, 5.8 \mu \mathrm{m}\right)$ and the $v_{3} \mathrm{CH}_{2}$ scissors $\left(1490 \mathrm{~cm}^{-1}, 6.7 \mu \mathrm{m}\right)$ vibrational bands have the highest 


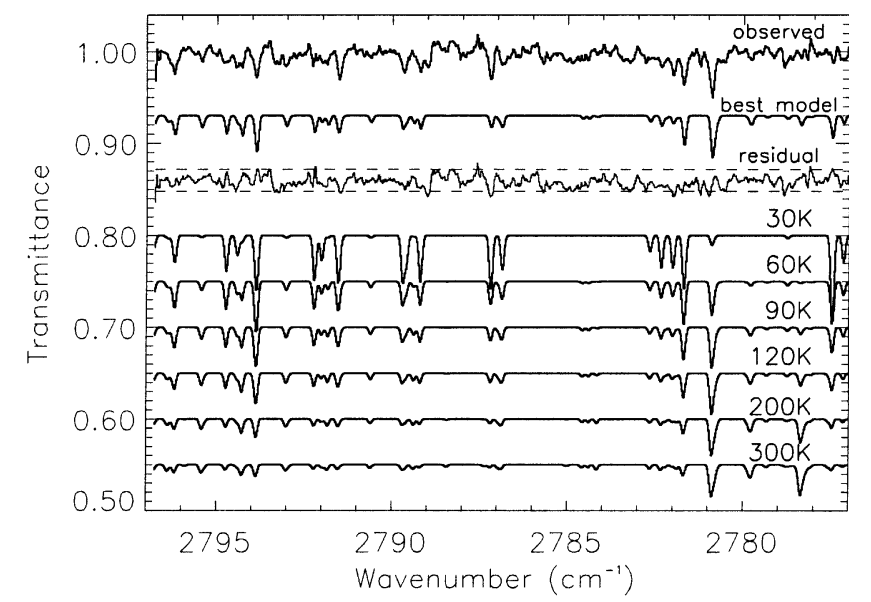

Fig. 2. Observed spectrum of W33A divided by the estimated local dust continuum to provide a transmittance spectrum, and model absorption spectra of $\mathrm{H}_{2} \mathrm{CO}$ at various excitation temperatures, for $N\left(\mathrm{H}_{2} \mathrm{CO}\right)=2 . \times 10^{16} \mathrm{~cm}^{-2}$. The best fit (see text) model spectrum and its residuals are shown just below the observed spectrum.

absorbance. Schutte et al. (1993) have measured a number of infrared bands of pure and mixed $\mathrm{H}_{2} \mathrm{CO}$ ices at $10 \mathrm{~K}$ in order to facilitate the identification of solid formaldehyde in the mantles present in circumstellar environments. The first "pre-ISO" observational attempts by Schutte et al. (1996) to identify solid phase $\mathrm{H}_{2} \mathrm{CO}$ in these environments were controversial, as their estimates were based on the $v_{1}$ and $v_{5} \mathrm{C}-\mathrm{H}$ stretching modes at 2865 and $2800 \mathrm{~cm}^{-1}$ which are observable from ground based telescopes but rather difficult to disentangle from absorptions due to solid methanol, which is abundant in the same sources. Subsequently the stronger $v_{2}$ and $v_{3}$ modes of solid $\mathrm{H}_{2} \mathrm{CO}$ were found by ISO towards a number of high mass protostellar envelopes (Keane et al. 2001; Dartois et al. 1999; Gibb et al. 2000). However, accurate determination of column densities of solid phase $\mathrm{H}_{2} \mathrm{CO}$ in the ISO spectra also is difficult, as the $\mathrm{H}_{2} \mathrm{CO}$ bands are blended with the dominant bending mode of $\mathrm{H}_{2} \mathrm{O}$ in the same wavelength region.

\section{Comparison with models}

Figure 2 displays the observed spectrum divided by the estimated local dust continuum, together with model spectra of the $v_{1}$ band of formaldehyde for a column density of $2 \times 10^{16} \mathrm{~cm}^{-2}$ at temperatures between 30 and $300 \mathrm{~K}$. The simulated spectra were calculated assuming a turbulent velocity of $8.6 \mathrm{~km} \mathrm{~s}^{-1}$, as measured by Mitchell et al. (1988) in CO high resolution FTS infrared absorption spectra towards W33A, and then convolved with a Gaussian function corresponding to the resolution of CGS4 ( $9 \mathrm{~km} \mathrm{~s}^{-1}$, i.e. $R \approx 33000$ ).

Although it is doubtful that all of the observed $\mathrm{H}_{2} \mathrm{CO}$ is at a single temperature, the model spectra at some of the intermediate temperatures fit the observed spectrum reasonably well. As can be seen in Fig. 2, the observed absence of the line at $2777.5 \mathrm{~cm}^{-1}$ and presence of the strong $2781 \mathrm{~cm}^{-1}$ line constrain the lower bound of temperatures, whereas the strengths of the lines in the $2782-2790 \mathrm{~cm}^{-1}$ interval relative to $2781 \mathrm{~cm}^{-1}$ rule out both the lowest and highest temperature model spectra. A $\chi^{2}$ analysis of the simulated and observed spectra indicates that the best fit is obtained from a mixture of formaldehyde at two different temperatures: $60 \%$ of the gas at a temperature of $90 \mathrm{~K}$ and $40 \%$ at $120 \mathrm{~K}$.

Thus, our simulations indicate that $N\left(\mathrm{H}_{2} \mathrm{CO}\right)=2 \pm$ $0.5 \times 10^{16} \mathrm{~cm}^{-2}$ (based mainly on the strongest lines in the $2780-2783 \mathrm{~cm}^{-1}$ interval) and a temperature of approximately $100 \mathrm{~K}$. Assuming a similar rotational excitation for RAFGL 7009S as W33A, we infer an upper limit to $N\left(\mathrm{H}_{2} \mathrm{CO}\right)$ of $8 \times 10^{15} \mathrm{~cm}^{-2}$. A higher signal-to-noise ratio spectrum of W33A would allow an analysis over a wider range of rotational levels and thus give better constraints on the column density and temperature. It is known that the temperature of much of the cloud in front of W33A is approximately $23 \mathrm{~K}$ (Mitchell et al. 1988). From the model spectra we estimate that column density of $\mathrm{H}_{2} \mathrm{CO}$ at this temperature cannot be more than about one-seventh of the above value, thus demonstrating that the observed formaldehyde is not uniformly distributed in the cloud. Formaldehyde has been observed in absorption in W33A at $6 \mathrm{~cm}$ (Goss et al. 1978) but no quantitative column density has been derived.

\section{Discussion}

Based on single dish and interferometric millimeter wave observations van der Tak et al. (2000) conclude that W33A is one of several high mass protostars which is surrounded by a volume a few arcseconds in size in which methanol abundances are two orders of magnitude higher than in the remainder of the cloud. The high temperature of the methanol and the size of the region suggests that the high abundance is due to evaporation of grain mantles. However, van der Tak et al. (2000) concluded that the $\mathrm{H}_{2} \mathrm{CO}$ that they observed toward W33A and elsewhere is roughly uniformly distributed within the large ( $\left.\gg 3^{\prime \prime}\right)$ beam of the single dish and therefore is not an immediate product of mantle evaporation. In contrast to this, Sutton et al. (1995) have found that the abundance of $\mathrm{H}_{2} \mathrm{CO}$ in the prototypical and nearest hot core, Orion/KL, is enhanced relative to its surroundings. Thus there may be considerable diversity in the compositions of cores in regions of massive star formation. In the material surrounding low mass protostars, analysis by Maret et al. (2004) and Schöier et al. (2004) of millimeter wave formaldehyde emission suggests a spatial jump in the $\mathrm{H}_{2} \mathrm{CO}$ abundance occuring when the dust temperature reaches $100 \mathrm{~K}$, the sublimation temperature of the grain mantles. The abundance rises from $\mathrm{H}_{2} \mathrm{CO}: \mathrm{H}_{2} \sim 10^{-10}-10^{-9}$ in the extended background to $\sim 10^{-8}-10^{-7}$ in the inner region in these low mass objects. These results are qualitatively similar to what is observed in Orion/KL but apparently are not at all similar to what van der Tak et al. (2000) found for other massive protostars. A more recent paper by Jørgensen et al. (2005) suggests that single dish observations do not require large $\mathrm{H}_{2} \mathrm{CO}$ abundance enhancements in the inner warm regions surrounding low mass protostars, but that such enhancements also cannot be ruled out. 
Table 2. Molecular gas phase observations.

\begin{tabular}{|c|c|c|c|c|c|c|c|}
\hline \multicolumn{2}{|l|}{ Source } & \multicolumn{3}{|c|}{ W33A } & \multicolumn{3}{|c|}{ RAFGL 7009S } \\
\hline \multirow[t]{2}{*}{ Molecule } & comment & Temperature & column density & Reference & Temperature & column density & Reference \\
\hline & & K & $10^{16} \mathrm{~cm}^{-2}$ & & $\mathrm{~K}$ & $10^{16} \mathrm{~cm}^{-2}$ & \\
\hline${ }^{13} \mathrm{CO}$ & IR absorption & $120_{-10}^{+14}$ & $23 \pm 4$ & 1 & & & \\
\hline${ }^{13} \mathrm{CO}$ & IR absorption & $23_{-4}^{+5}$ & $21 \pm 9$ & 1 & & & \\
\hline $\mathrm{H}_{2}{ }^{\text {hot }}$ & from ${ }^{13} \mathrm{CO}$ & 120 & $6.9 \times 10^{6}$ & 4 & & $5.2 \times 10^{7}$ & 7 \\
\hline & for W33A & & & & & & \\
\hline $\mathrm{H}_{2}{ }^{\text {cold }}$ & from ${ }^{13} \mathrm{CO}$ & 23 & $6.3 \times 10^{6}$ & 4 & & & \\
\hline & for W33A & & & & & & \\
\hline $\mathrm{CO}_{2}$ & IR absorption & $100_{-30}^{+50}$ & $3.2 \pm 1.0$ & 2 & & & \\
\hline $\mathrm{CH}_{4}$ & IR absorption & $110 \pm 15$ & $11.1 \pm 2.4$ & 3 & & & \\
\hline $\mathrm{HCN}$ & IR absorption & $80_{-40}^{+100}$ & $0.3 \pm 0.2$ & 4 & & & \\
\hline $\mathrm{H}_{2} \mathrm{O}$ & IR absorption & 120 (from $\mathrm{CO}$ ) & $\leq 80$ & 5 & & & \\
\hline $\mathrm{C}_{2} \mathrm{H}_{2}$ & IR absorption & 10 & $0.5 \pm 0.2$ & 4 & & & \\
\hline $\mathrm{H}_{2} \mathrm{CO}$ & IR absorption & 90 & $2 \pm 0.5$ & present work & $90^{*}$ & $\leq 0.8$ & present work \\
\hline $\mathrm{H}_{2} \mathrm{CO}$ & IR absorption & 23 & $<0.3 \pm 0.5$ & present work & & & \\
\hline $\mathrm{H}_{2} \mathrm{CO}$ & submm emission & 88 & 0.012 & 6 & & - & 6 \\
\hline $\mathrm{CH}_{3} \mathrm{OH}$ & mm emission & 155 & 0.2 & 6 & 8 & 0.39 & 6 \\
\hline
\end{tabular}

1: Mitchell et al. (1990), 2: Boonman et al. (2003), 3: Boogert et al. (1998), 4: Lahuis \& van Dishoeck (2000), 5: Boonman \& van Dishoeck (2003), 6: van der Tak et al. (2000), 7: Dartois et al. (2000). ${ }^{*}$ The same temperature as W33A is assumed.

\subsection{Location and abundance}

Table 2 summarizes information on the gas phase molecular content toward W33A and RAFGL 7009S derived from infrared absorption and submillimeter emission spectroscopy. The excitation temperatures of most of the molecules detected in absorption toward W33A are in the $80-120 \mathrm{~K}$ range (except acetylene for which the excitation temperature is $10 \mathrm{~K}$ ). The excitation temperatures of millimeter wave-emitting formaldehyde and methanol are roughly in the same range. However, the column density of the millimeter wave-emitting $\mathrm{H}_{2} \mathrm{CO}$, which is an average over a vastly larger beam $\left(\sim 15^{\prime \prime}\right)$ than that defined by the sub-arcsecond size of the infrared continuum source at $3.6 \mu \mathrm{m}$, is two orders of magnitude smaller than the column density of $\mathrm{H}_{2} \mathrm{CO}$ observed in absorption at $3.6 \mu \mathrm{m}$. The same remark also applies to the methanol emission spectra. Thus the reported column densities of $\mathrm{H}_{2} \mathrm{CO}$ by van der Tak et al. (2000) are not easily applicable to our absorption measurements which were made along a pencil beam to an embedded and pointlike infrared source. However, it is clear that the formaldehyde which we have observed in absorption is strongly concentrated close to the W33A continuum source.

The column density of "hot" $\mathrm{H}_{2} \mathrm{CO}$ and the upper limit on "cold" $\mathrm{H}_{2} \mathrm{CO}$ estimated in Sect. 4 can be compared to the column densities of "hot" and "cold" molecular hydrogen derived from $\mathrm{CO}$ observations, to give formaldehyde abundances. These are respectively $\sim 3 \times 10^{-7}$ and $<5 \times 10^{-8}$, respectively. The upper limit derived for RAFGL 7009S corresponds to $\mathrm{H}_{2} \mathrm{CO}: \mathrm{H}_{2} \leq 1.7 \times 10^{-8}$. The value derived for $\mathrm{W} 33 \mathrm{~A}$ is at the upper end of the above jump models, whereas the limit for RAFGL 7009S is non-constraining.

\subsection{Evaporation or gas phase chemistry?}

Observations of high mass star-forming regions provide an opportunity to determine the gas to solid ratio for a variety of molecular species. These ratios can shed light on the chemistry taking place, physical processes involved, and evolutionary states of the protostars. We give in Table 3 the column densities and the gas to solid ratio for a number of molecular species observed toward W33A and (where observed) RAFGL 7009S. The ratios do not correspond to any particular region in the cloud, as most of the gaseous species are located in a warm environment whereas the ices can only exist at much lower temperatures.

The low gas-to-solid ratios and high temperature of the gaseous $\mathrm{H}_{2} \mathrm{CO}$ are qualitatively consistent with the formaldehyde being produced by evaporating mantles in a small fraction of the cloud. If the gas phase species come directly from evaporation of the ices (i.e., timescales for abundance changes due to chemistry are slower than the fresh evaporation timescale), one would expect similar patterns of abundance ratios in the gas and solid phases relative to a common species. To test this we compare in Table 4 the abundances relative to warm $\mathrm{CO}$ of formaldehyde, methanol, methane, and carbon dioxide toward W33A where substantial information is available. The column density of hot $\mathrm{CO}$ is obtained from the value for ${ }^{13} \mathrm{CO}$ in Table 2 assuming an isotopic ratio of 60 , so that $N($ CO-hot $)=1.4 \times 10^{19} \mathrm{~cm}^{-2}$. For RAFGL 7009S the table only includes abundance ratios relative to $\mathrm{CO}$ of the ice mantles, as the corresponding gas phase information is not available.

The table shows that in W33A the abundance ratios relative to $\mathrm{CO}$ are drastically different in the gas and solid phases, 
Table 3. Column densities of ices and gas-to-solid column density ratios toward W33A and RAFGL 7009S.

\begin{tabular}{ccccccc}
\hline \hline $\begin{array}{c}\text { Source } \\
\text { Molecule } \\
\text { in solid phase }\end{array}$ & $\begin{array}{c}\text { Column density } \\
10^{17} \mathrm{~cm}^{-2}\end{array}$ & $\begin{array}{c}\text { W33A } \\
\text { Reference }\end{array}$ & Gas/Solid & $\begin{array}{c}\text { RAFGL 7009S } \\
\text { Column density } \\
10^{17} \mathrm{~cm}^{-2}\end{array}$ & Reference & Gas/Solid \\
\hline $\mathrm{CO}$ & $8.9 \pm 0.5$ & 1 & 0.26 & 18 & 4 & \\
$\mathrm{CO}_{2}$ & $14.5 \pm 1.3$ & 1 & 0.022 & $25 \pm 1.3$ & 2 & \\
$\mathrm{CH}_{4}$ & $1.7 \pm 0.2$ & & 0.65 & 4.3 & 2 & \\
$\mathrm{HCOOH}$ & 1.8 & 3 & & $5.2 \pm 1.1$ & 1 & \\
$\mathrm{H}_{2} \mathrm{O}$ & 110 & 1 & $\leq 0.07$ & 110 & 4 & $\geq 0.18$ \\
$\mathrm{H}_{2} \mathrm{CO}$ & 7.1 & 3 & $\sim 0.03$ & 3.3 & 4 & $\leq 0.02$ \\
$\mathrm{CH}_{3} \mathrm{OH}$ & $17 \pm 4$ & 3 & $\sim 0.19^{a}$ & 33 & 3 & \\
\hline
\end{tabular}

1: Gibb et al. (2004), 2: Dartois et al. (1998), 3: Keane et al. (2001), 4: Dartois et al. (1999).

${ }^{a}$ Estimated from the measured column density of $\mathrm{CH}_{3} \mathrm{OH}$ in emission at millimeter wavelengths scaled by the ratio of column densities of $\mathrm{H}_{2} \mathrm{CO}$ in IR absorption and millimeter wave emission: $N\left(\mathrm{CH}_{3} \mathrm{OH}_{\text {absorption }}\right) \sim 3.3 \times 10^{17} \mathrm{~cm}^{-2}$.

Table 4. Abundances relative to gaseous and frozen CO.

\begin{tabular}{|c|c|c|c|}
\hline \multirow{2}{*}{$\begin{array}{c}\text { Source } \\
\text { Molecule }\end{array}$} & \multicolumn{2}{|c|}{ W33A } & \multirow{2}{*}{$\begin{array}{c}\text { RAFGL 7009S } \\
\text { Ice mantle }\end{array}$} \\
\hline & Gas phase & Ice mantle & \\
\hline $\mathrm{H}_{2} \mathrm{CO}$ & $1.4 \times 10^{-3}$ & 0.8 & 0.18 \\
\hline $\mathrm{CH}_{3} \mathrm{OH}$ & $2.4 \times 10^{-2 a}$ & 1.9 & 1.8 \\
\hline $\mathrm{CH}_{4}$ & $7.8 \times 10^{-3}$ & 0.19 & 0.24 \\
\hline $\mathrm{CO}_{2}$ & $2.3 \times 10^{-3}$ & 1.63 & 1.4 \\
\hline
\end{tabular}

whereas the abundance ratios in the ice mantles are fairly similar in the two sources. This is not surprising, as CO is the most volatile of the species in mantles. However, in W33A the table also reveals quite different relative abundances of other species in the two phases; for example, methanol is 2.4 times more abundant than formaldehyde in the W33A ice mantles, but is 16 times more abundant in the gas phase (both from predicted infrared and actual millimeter measurements; see Table 2). This indicates that gas phase chemical processing alters the abundances of some of the species, and thus that even in these less volatile species observations of the gas do not provide a direct picture of the ice content.

\subsection{Physical processes and formaldehyde production}

Photoionization of the near stellar environment around a young massive star produces a compact or ultra-compact HII region which is thought to exist for $\sim 10^{5}$ years after the formation of the massive star (Ellingsen et al. 2005). W33A and RAFGL 7009S appear to be in this phase. Weak $\mathrm{Br} \alpha$ line emission has been detected from W33A (McGregor et al. 1984). No hydrogen recombination lines have been detected toward RAFGL 7009S (Dartois et al. 2003), but the source does have detectable radio continuum (McCutcheon et al. 1995), as does W33A (Rengarajan \& Ho 1996). W33A and especially RAFGL 7009S probably are in the early stages of developing photoionising fronts and photodissociation regions; the outflow dynamical time scale of RAFGL $7009 \mathrm{~S}$ is $1.8 \times 10^{4} \mathrm{yr}$ (Shepherd \& Churchwell 1996). The HII regions in these objects probably are still squashed against the photospheres of the exciting stars by the accreting gas and the hot cores in these objects may be in their earliest stages of development. In $\mathrm{W} 33 \mathrm{~A}$, release of solid formaldehyde into the gas phase has probably not occurred in the entire volume over which the submillimeter emission lines of formaldehyde are detected; in RAFGL $7009 \mathrm{~S}$ if a region of enhanced $\mathrm{H}_{2} \mathrm{CO}$ exists, either the enhancement or the volume is considerably smaller than in W33A. In both cases the volume of matter exposed to the fronts is probably very small, due to the high density near where the massive protostar has formed.

The $\mathrm{H}_{2} \mathrm{CO}$ that we have detected toward W33A is not directly exposed to the UV flux of the central O6-O7 star, as the continuum, on which are seen the gas phase $\mathrm{H}_{2} \mathrm{CO}$ absorption lines, is produced by dust grains. The grains reprocess the ultraviolet and visible radiation from the protostar into infrared continuum radiation and shield the formaldehyde and other molecules from photodissociation. Provided that these grains are not destroyed in a time comparable to formaldehyde photodissociation rates, the $\mathrm{H}_{2} \mathrm{CO}$ lifetime against photodissociation is determined by the attenuation by envelope out to a radius where $T \approx 100 \mathrm{~K}$ (several magnitudes of visual extinction). Using the lifetimes of $\mathrm{H}_{2} \mathrm{CO}$ against photodissociation estimated by Aiello et al. (1981), with an impinging field scaled to the luminosity of $\mathrm{W} 33 \mathrm{~A}$, we find values in the range $10^{2}-10^{4}$ years. If $\mathrm{H}_{2} \mathrm{CO}$ and $\mathrm{CH}_{3} \mathrm{OH}$ evaporate from the grain mantles and are mainly photochemically destroyed, one should expect the ratio of hot $(100 \mathrm{~K}) \mathrm{H}_{2} \mathrm{CO}$ to hot $\mathrm{CH}_{3} \mathrm{OH}$ to reflect to first order the solid abundances in this inner region, i.e. to be $40 \%$ for $\mathrm{W} 33 \mathrm{~A}$, as the photodissociation rates of methanol and formaldehyde are of the same order (Roberge et al. 1991). However, as pointed out above, the ratio from millimeter gas phase emission, $\sim 6 \%$, is much lower.

Ultraviolet photons that leak through the dust and impinge on gas phase methanol could in principle contribute to the formation of formadehyde. Ultraviolet photolysis of methanol, which requires photon energies $\geq 4.5 \mathrm{eV}$, yields the metoxy radical $\left(\mathrm{CH}_{3} \mathrm{O}\right)$ as the dominant channel (Wen et al. 1994; Cheng et al. 2002), whereas the threshold energy for ionization of 
methanol is $11 \mathrm{eV}$. Photodissociation of formaldehyde itself (at energies $\geq 3.8 \mathrm{eV}$ ) restricts the efficiency of its formation. These chemical pathways can either proceed through the preexisting gas responsible for the extended background of $\mathrm{H}_{2} \mathrm{CO}$ or via the products released by sublimating ices.

Recently Stäuber et al. (2004) have considered the role of UV radiation from a massive young stellar object on the chemistry of its envelope, but do not report results for formaldehyde. As atomic oxygen is predicted to be abundant and not very dependent on the extent of the UV flux, $\mathrm{O}+\mathrm{CH}_{3} \rightarrow \mathrm{H}_{2} \mathrm{CO}+\mathrm{H}$ is potentially an efficient source of formaldehyde. An alternative gas phase route is provided by $\mathrm{OH}+\mathrm{CH}_{2} \rightarrow \mathrm{H}_{2} \mathrm{CO}+\mathrm{H}$, but it is probably less efficient as the predicted column density of $\mathrm{OH}$ is about 100 times less than that of atomic oxygen. However, the temperatures where reactions between atoms and radicals are important in the Stauber model are higher than those where the $\mathrm{H}_{2} \mathrm{CO}$ where the $\mathrm{H}_{2} \mathrm{CO}$ absorption lines are formed.

The two zone model developed by Doty et al. (2002) for another massive object (AFGL 2591) is in qualitative agreement with the above description of a relatively shielded environment. In the inner zone $(T>100 \mathrm{~K})$ where Doty et al. (2002) set the initial abundances of $\mathrm{H}_{2} \mathrm{CO}$ and $\mathrm{CH}_{3} \mathrm{OH}$ to $1.2 \times 10^{-7}$ and $10^{-6}$, respectively, the fractional abundances do not vary significantly during $3 \times 10^{5}$ years, thus preserving the initial conditions that could be set by thermal evaporation of ices (see their Fig. 12). In the outer zone $(T<100 \mathrm{~K})$, where methanol and formaldehyde initial abundances were depleted by freeze out, gas phase chemistry is able to produce amounts of $\mathrm{H}_{2} \mathrm{CO}$ in agreement with extended sources probed by submillimeter observations, but not $\mathrm{CH}_{3} \mathrm{OH}$. Only a strong heating event is able to destabilise the initial abundances set in the inner zones. At the evolutionary stages of the embedded W33A and RAFGL 7009S sources, formaldehyde formation seems to be driven by the balance between thermal ice evaporation and the efficiency of penetration of the disrupting front providing higher temperature gas phase chemistry inside the envelope.

\section{Summary and conclusions}

We have detected infrared absorption by gas phase formaldehyde towards W33A, the first such detection in any astronomical object. Comparison with millimeter wave observations of the same species strongly implies that the gaseous formaldehyde is strongly concentrated at the infrared continuum source. The formaldehyde gas-to-ice ratio is about 0.03 and the abundance relative to molecular hydrogen is about $3 \times 10^{-7}$. The derived mean temperature of the $\mathrm{H}_{2} \mathrm{CO}$ is $\sim 100 \mathrm{~K}$, similar to the temperature of most other gas phase molecules observed towards this source, and is approximately the temperature at which ice mantle matrices sublime. Although some of the observed $\mathrm{H}_{2} \mathrm{CO}$ may come directly from the solid state, the large differences of the relative abundances of $\mathrm{H}_{2} \mathrm{CO}$ and other molecular species between mantles and the gas phase suggests that other physical processes must alter the gas phase abundances. Photodissociation of the thermally released ices, $\mathrm{H}_{2} \mathrm{O}$, $\mathrm{CH}_{3} \mathrm{OH}, \mathrm{CH}_{4}$, and $\mathrm{CO}$ and $\mathrm{H}_{2} \mathrm{CO}$ can provide chemical pathways that change the abundances.
Follow-up studies from the ground at the highest possible spectral resolution are needed to derive more constraints on the chemical processes at work. In particular, the kinematic structure of infrared absorption lines might allow one to determine the exact location and the origin of the observed formaldehyde. A similar infrared absorption study of gas phase methanol is highly desirable but a quantitative analysis depends on the availability of detailed spectroscopic data for analysis of the methanol infrared vibrational modes.

Acknowledgements. We thank the staff of the United Kingdom Infrared Telescope, which is operated by the Joint Astronomy Centre on behalf of Particle Physics and Astronomy Research Council (PPARC). We are grateful to Agnes Perrin who calculated for us the positions and intensities of the $\mathrm{Q}$ branch of the $v_{2}$ mode of $\mathrm{H}_{2} \mathrm{CO}$, to Takeshi Oka for a number of helpful comments, and to the referee for constructive suggestions. T.R.G.'s research is supported by the Gemini Observatory, which is operated by the Association of Universities for Research in Astronomy, Inc., on behalf of the international Gemini partnership of Argentina, Australia, Brazil, Canada, Chile, the United Kingdom and the United States of America.

\section{References}

Aiello, S., Morbidelli, L., \& Ulivi, L. 1981, Ap\&SS, 80, 173

Boogert, A. C. A., Helmich, F. P., van Dishoeck, E. F., et al. 1998, A\&A, 336, 352

Boogert, A. C. A., Blake, G. A., \& Öberg, K. 2004, ApJ, 615, 344

Boonman, A. M. S., \& van Dishoeck, E. F. 2003, A\&A, 403, 1003

Boonman, A. M. S., van Dishoeck, E. F., Lahuis, F., \& Doty, S. D. 2003, A\&A, 399, 1063

Bronfman, L., Nyman, L.-A., \& May, J. 1996, A\&AS, 115, 81

Brown, L. R., Hunt, R. H., \& Pine, A. S. 1979, J. Mol. Spectr., 75, 406

Cheng, B., Bahou, M., Chen, W., et al. 2002, J. Chem. Phys., 117, 1633

Dartois, E., D’Hendecourt, L., Boulanger, F., et al. 1998, A\&A, 331, 651

Dartois, E., Gerin, M., \& d'Hendecourt, L. 2000, A\&A, 361, 1095

Dartois, E., Schutte, W., Geballe, T. R., et al. 1999, A\&A, 342, L32

Dartois, E., Thi, W.-F., Geballe, T. R., et al. 2003, A\&A, 399, 1009

Disanti, M. A., dello Russo, N., Magee-Sauer, K., et al. 2002, in ESA SP-500: Asteroids, Comets, and Meteors: ACM 2002, 571

Doty, S. D., van Dishoeck, E. F., van der Tak, F. F. S., \& Boonman, A. M. S. 2002, A\&A, 389, 446

Ellingsen, S. P., Shabala, S. S., \& Kurtz, S. E. 2005, MNRAS, 357, 1003

Geballe, T. R., \& Oka, T. 1996, Nature, 384, 334

Gibb, E. L., Whittet, D. C. B., Boogert, A. C. A., \& Tielens, A. G. G. M. 2004, ApJS, 151, 35

Gibb, E. L., Whittet, D. C. B., Schutte, W. A., et al. 2000, ApJ, 536, 347

Goss, W. M., Matthews, H. E., \& Winnberg, A. 1978, A\&A, 65, 307

Herndon, S. C., Nelson, D. D., Li, Y., \& Zahniser, M. S. 2005, J. Quant. Spectr. Radiat. Transf., 90, 207

Jørgensen, J. K., Schöier, F. L., \& van Dishoeck, E. F. 2005, A\&A, 437, 501

Keane, J. V., Tielens, A. G. G. M., Boogert, A. C. A., Schutte, W. A., \& Whittet, D. C. B. 2001, A\&A, 376, 254

Knacke, R. F., Brooke, T. Y., \& Joyce, R. R. 1986, ApJ, 310, L49

Lahuis, F., \& van Dishoeck, E. F. 2000, A\&A, 355, 699 
Maier, W. B., Holland, R. F., Freund, S. M., \& Beattie, W. H. 1980, J. Chem. Phys., 72, 264

Maret, S., Ceccarelli, C., Caux, E., et al. 2004, A\&A, 416, 577

McCall, B. J., Geballe, T. R., Hinkle, K. H., \& Oka, T. 1999, ApJ, 522, 338

McCutcheon, W. H., Sato, T., Purton, C. R., Matthews, H. E., \& Dewdney, P. E. 1995, AJ, 110, 1762

McGregor, P. J., Persson, S. E., \& Geballe, T. R. 1984, PASP, 96, 315

Mitchell, G. F., Allen, M., \& Maillard, J. 1988, ApJ, 333, L55

Mitchell, G. F., Maillard, J., Allen, M., Beer, R., \& Belcourt, K. 1990, ApJ, 363, 554

Mumma, M. J., \& Reuter, D. C. 1989, ApJ, 344, 940

Perrin, A., Keller, F., \& Flaud, J.-M. 2003, J. Mol. Spectr., 221, 192

Rengarajan, T. N., \& Ho, P. T. P. 1996, ApJ, 465, 363

Roberge, W. G., Jones, D., Lepp, S., \& Dalgarno, A. 1991, ApJS, 77, 287
Rothman, L. S., Barbe, A., Benner, D. C., et al. 2003, J. Quant. Spectr. Radiat. Transf., 82, 5

Schöier, F. L., Jørgensen, J. K., van Dishoeck, E. F., \& Blake, G. A. 2004, A\&A, 418, 185

Schutte, W. A., Allamandola, L. J., \& Sandford, S. A. 1993, Icarus, 104,118

Schutte, W. A., Gerakines, P. A., Geballe, T. R., van Dishoeck, E. F., \& Greenberg, J. M. 1996, A\&A, 309, 633

Shepherd, D. S., \& Churchwell, E. 1996, ApJ, 457, 267

Stäuber, P., Doty, S. D., van Dishoeck, E. F., Jørgensen, J. K., \& Benz, A. O. 2004, A\&A, 425, 577

Sutton, E. C., Peng, R., Danchi, W. C., et al. 1995, ApJS, 97, 455

van der Tak, F. F. S., van Dishoeck, E. F., \& Caselli, P. 2000, A\&A, 361,327

Wen, Y., Segall, J., Dulligan, M., \& Wittig, C. 1994, J. Chem. Phys., 101,5665 